\title{
Microarray tools to unveil viral-microbe interactions in nature
}

\author{
Fernando Santos ${ }^{1}$, Manuel Martínez-García ${ }^{1}$, Víctor Parro ${ }^{2}$ and Josefa Antón ${ }^{1}$ * \\ 1 Departamento de Fisiología, Genética y Microbiología, Universidad de Alicante, Alicante, Spain \\ 2 Departamento de Evolución Molecular, Centro de Astrobiología (INTA-CSIC), Madrid, Spain
}

\section{Edited by:}

Heather K. Allen, National Animal

Disease Center, USA

Reviewed by:

Loren John Hauser, University of

Tennessee Knoxville, USA

Carl James Yeoman, Montana State

University, USA

*Correspondence:

Josefa Antón, Departamento de

Fisiología, Genética y Microbiología,

Universidad de Alicante, Apartado

99, 03080 Alicante, Spain

e-mail: anton@ua.es
The interactions between viruses and their microbial hosts play a central role in the control of microbial communities in nature. However, the study of such interactions within the uncultured majority is technically very challenging. Here, we review how microarray tools can be used to analyze the interactions between viruses and their microbial hosts in nature, away from laboratory pure culture-based models. We show examples of how DNA arrays have been used to study the expression of viral assemblages in natural samples, and to assign viruses to hosts within uncultured communities. Finally, we briefly discuss the possibilities of protein and glycan arrays to gain insight into the ways microbes interact with their viruses.

Keywords: phage-bacteria interactions, microarray analysis, virus ec, microbiology, virus-, virus-host interaction

\section{INTRODUCTION}

As stated in the editorial of this issue of Frontiers in Microbiology, the nature of life is change (Allen and Abedon, 2013). Changes occur at every level of biological complexity and are especially fast and dramatic within the microbial world. These changes affect the complex networks of interactions between viruses and their hosts that structure microbial communities in nature. Here, we review how microarrays can be used to analyze the interactions between uncultured viruses and their microbial hosts in nature, away from laboratory pure culture-based models. In addition to providing some examples on the successful use of this technology, we offer some ideas about its potential applications.

Microarray technology was developed in the mid-1990s (Schena et al., 1995). Microarrays/chips are created by the immobilization of molecules in discrete spatial locations (Figure 1), normally at high density, on a solid surface. Depending on the nature of the molecule immobilized on the physical substrate, there are different types of microarrays, such as DNA, protein, glycan, small molecule, cell, or even tissues (Berard et al., 2012). The power of microarrays lies in their unprecedented capacity to simultaneously interrogate tens to hundreds of thousands of immobilized probes.

Microchips have been used extensively to study viral biology, many times addressing ecologically relevant questions. Most of these studies are circumscribed to the analysis of isolated viruses or to issues related to the role of viruses in human health and would therefore not fall within the scope of this mini review. However, many of the examples of microarray applications in the field of human virology could be (or have been) "upscaled" from individual populations to the whole viral community present in the samples under study. A similar rationale boosted the birth of microbial metagenomics, which benefited from the sequencing of the human genome.

The first uses (Table 1) of microarray tools in microbial viral ecology focused on the description of the diversity and dynamics of viral assemblages in environmentally relevant isolated viralhost pairs. In these examples, DNA probes encompassing the complete viral genomes were immobilized on a chip and used to interrogate their expression within infected cells or to conduct viral comparative genomic studies of isolated viruses.

Combined with metagenomics, microarrays have been used to analyze viral diversity and dynamics within natural samples (Table 1). For instance, Breitbart et al. (2008) constructed custom arrays with sequences retrieved from a week-old infant virome to follow the evolution of the viral assemblage during the first 2 weeks of the infant life. More recently, in a very elegant application of microarrays in viral ecology, Snyder et al. (2010) used a cellular CRISPR spacer-based microarray for detection of viruses in Archaea-dominated acidic hot springs samples, taken from Yellowstone National park (USA). The idea behind the design is that the spacers contained within the direct repeat units in the CRISPR loci of cellular genomes often derive from viruses that infected the cell in the past. Therefore, the spacer sequences are in a way a record of the infection history of the cell. These sequences were retrieved from environmental cellular metagenomes (by in silico search and PCR amplification) and immobilized as probes into a chip to interrogate the viral assemblage. This array proved to be useful both in the detection of unknown viruses and in the monitoring of viral dynamics within the community.

Nowadays, given the lower costs and relative ease of Next Generation Sequencing techniques, microarrays are no longer needed for addressing the issues mentioned above. However, this approach still offers some unparalleled possibilities, as described below.

\section{METAVIROTRANSCRIPTOMIC STUDIES}

Array-based viral metatranscriptomic studies overcome the fact that there is no straightforward, unambiguous way to extract viral mRNA from bulk mRNAs and make the sequencing of all mRNAs unnecessary. Thus, using the same terms presented in 


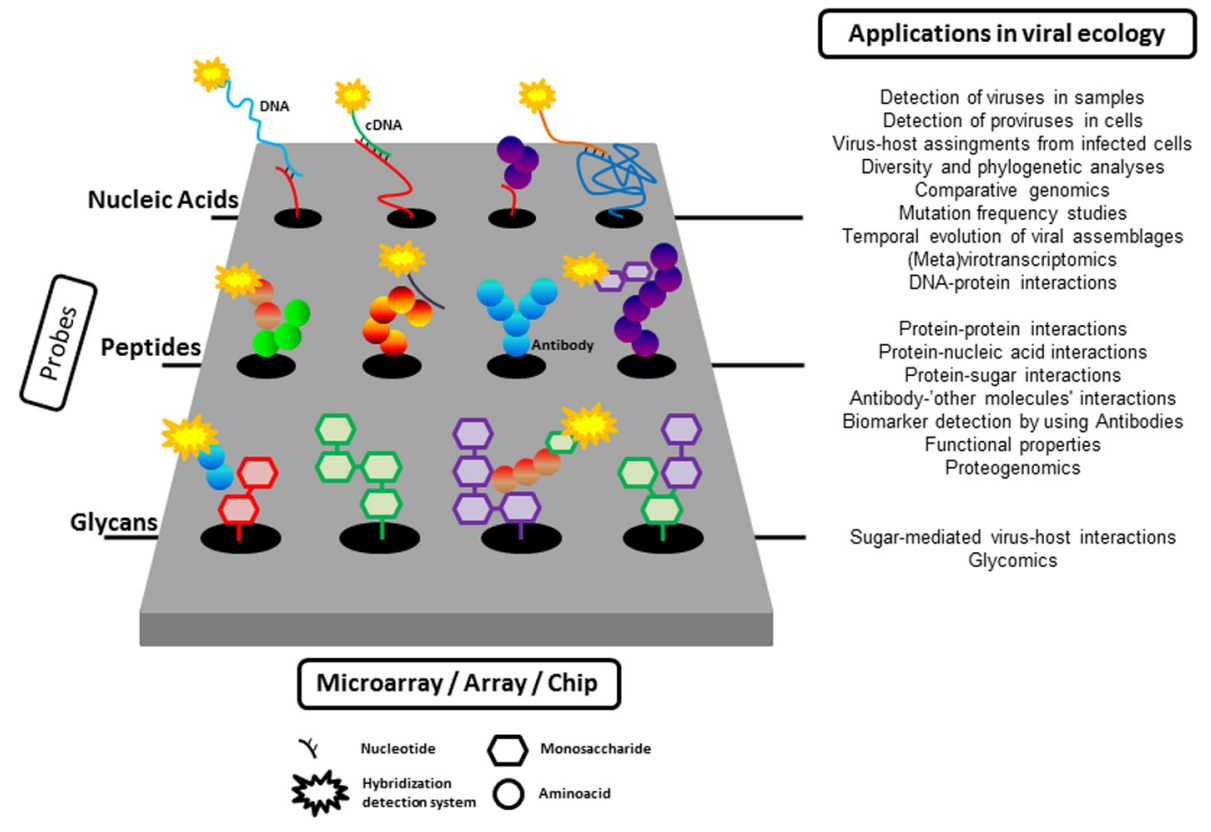

FIGURE 1 | A summary of the application of microarray tools to the study of viral ecology. As described in the text, nucleic acids (from oligonucleotide to complete viral genomes), proteins, or glycans can be immobilized on a solid surface to probe different target molecules (nucleic acids, glycans, proteins). The interaction between immobilized probes and targets is normally detected by fluorescence although other detection systems are also available.

Table 1 | Some examples of the use of microarrays to study microbial viral ecology ${ }^{\mathrm{a}}$.

\begin{tabular}{|c|c|c|}
\hline Virus-host system/samples analyzed & Issue addressed & References \\
\hline GTAs $^{\mathrm{C}}$ and Rhodobacter capsulatus & Encapsulation of GTAs & Hynes et al., 2012 \\
\hline Coccolithoviruses and Emiliania huxleyi & Viral diversity and gene expression during infection & Allen and Wilson, 2008 \\
\hline Cyanophages and Prochlorococcus & Phage and host transcriptome dynamics & Lindell et al., 2007 \\
\hline Feces of new borns & Diversity and dynamics of the viral assemblage & Breitbart et al., 2008 \\
\hline Archaea-dominated acidic hot spring samples & Identification of CRISPRs spaces within the viral metagenome & Snyder et al., 2010 \\
\hline Candidatus Accumulibacter phosphatis- enriched sludge & Phage expression and dynamics & Kunin et al., 2008 \\
\hline Hypersaline sample & Expression of the viral assemblage & Santos et al., 2011 \\
\hline Archaea-dominated hypersaline samples & Identification of viral-hosts pairs & $\begin{array}{l}\text { Martínez-García et al., } \\
\text { in press }\end{array}$ \\
\hline
\end{tabular}

${ }^{a}$ There are many examples on the study of phage-bacteria interactions related to disease and food production that are not considered here. All the examples provided use different kinds of DNA microchip, except that of Barr et al. which uses a glycoarray (see text).

${ }^{b}$ Although this study could not be strictly considered as "microbial viral ecology," we have included it here because it is a widely used model of interaction between phage and bacterial host.

${ }^{c}$ Gene Transfer Agents.

Allen and Wilson (2008) it no longer seems likely that microarrays will be used to ask "who is there?" in the field of viral ecology, although they can definitely help to answer the ecologically relevant questions "what is a particular component of the community doing and how is it doing it?"

Kunin et al. (2008) analyzed viral and microbial assemblages in two geographically distant sludge bioreactors (one in the
USA, and one in Australia) that were enriched with a single bacterial species Candidatus Accumulibacter phosphatis (CAP). Comparison of viral and microbial metagenomes suggested that phages were active in the sludge ecosystem. To confirm this, the US sludge was monitored at three time points spanning 3 months using expression arrays constructed from selected predicted genes from both phage and bacterial metagenomes. Hybridization of 
cDNA from the three time-course points with the array indicated that a large number of viral genes were highly expressed and that some phages persisted for long periods in the sludge. These results indicated that the bacterial community in the sludge was under "persistent local predation pressure." Although microarrays had been used previously for studying the activity of viruses infecting humans, this is the first example of a metatranscriptomic approach specifically targeting viral mRNAs since, normally, in metatranscriptomic studies both viral and microbial mRNA are analyzed together and separated only in silico. As the authors state, this work illustrated the value of combining sequence and gene expression data from the bacterial and viral fractions to address ecologically relevant questions.

Applying a similar rationale, we analyzed the viral expression in one hypersaline environment using a metatranscriptomic approach in which clones from a metaviromic library were immobilized on a microarray and used as probes against total mRNA extracted from the hypersaline community (Santos et al., 2011). The immobilized metaviromic library had been prepared previously by cloning sheared viral DNA into plasmids. The metaviromic sequences were analyzed and classified according to their putative hosts based on GC content and dinucleotide frequency analysis, and thus each clone in the array corresponded to a genomic fragment of a virus tentatively assigned to a microbial host. The array was then hybridized with bulk mRNA extracted from the natural assemblage (previously converted into cDNA), which contained all the RNA from the microbial genomes expressed at the time of sampling as well as the viral RNA produced during infection. We found that the viral groups that had the highest hybridization signal in the array (i.e., highest expression levels compared to the rest of immobilized viruses) were those related to high GC content haloarchaea and Salinibacter representatives, which were minor components in the environment. In addition, we analyzed the changes in expression of the immobilized viruses when the natural samples were subjected to two stress conditions (ultraviolet radiation and dilution). More specifically, we also analyzed the expression of some viral genome fragments carrying non-synonymous single nucleotide polymorphisms (SNPs) and saw that differences in sequences could be related to changes in expression levels under various analyzed conditions. We suggested that the viral assemblages could include very closely related viruses, for which we proposed the term "ecoviriotypes," which would respond differentially to changes in environmental parameters.

\section{ASSIGNING VIRUSES TO HOSTS}

Quite recently, we combined microarrays with single cell genomics (SCG) to assign virus-host pairs within natural uncultured microbial communities without the need for culture techniques (Martínez-García et al., in press). We investigated virus-host pairs within an Archaea-dominated hypersaline sample. First, we constructed a fosmid viral metagenomic library. This cloning approach was chosen because the optimum insert size for fosmids (i.e., between 30 and $45 \mathrm{~kb}$ ) corresponded to the size of most haloviral genomes detected in the analyzed samples. Then, individual cloned viral genomes were immobilized in an array or "virochip" (which, in a way, resembles the one designed by
Wang et al., 2002, that contained probes targeting different families of human pathogenic viruses). In parallel, genomic contents of uncultured individual cells present in the sample were retrieved by fluorescence activated cell sorting and their genomes amplified by multiple displacement amplification. Finally, individual cell genomes were hybridized against the virochip. Infected cells and immobilized viruses yielding positive hybridization were then sequenced and characterized. With this new approach, we described the first uncultured virus infecting the ubiquitous, uncultured Nanohaloarchaeal group in hypersaline environments, demonstrating the usefulness of combining these two high-throughput technologies (microarrays and SCG).

The advances of SCG as a mean to decipher genetic information of uncultured cells is spurring the development of single virus genomics (Allen et al., 2011) for unveiling genomic viral diversity in nature, one virus at a time. So, following the same rationale as our above mentioned study, massive microarrays could be constructed with thousands of immobilized single viral and prokaryote genomes, as a way to assign viruses to hosts in the majority of uncultured microbes, disentangling the complex virus-host network interactions within uncultured assemblages.

\section{PROTEIN AND GLYCAN ARRAYS}

Protein arrays can be either analytical (to check for the presence of given proteins in the analyzed samples) or functional (to query for properties of the immobilized proteins) (Uzoma and Zhu, 2013). They can range from immobilized peptides to more complex systems as yeast two hybrid or phage display arrays. Analytical protein microarrays have been used for biomarker detection in virus infection, in the search for novel serological biomarkers in disease, and in the study of lectin-glycan interactions to characterize mammalian cell envelopes. Functional protein microarrays can be used for identifying protein-protein, protein-lipid, proteinantibody, protein-small molecule, protein-DNA (transcription factors), protein-RNA, protein-lectin interactions; for identifying substrates or enzymes corresponding to different modifications; and for profiling the immune response (reviewed in Hsu and Mahal, 2009; Ben-Ari et al., 2013; Uzoma and Zhu, 2013).

One specific type of protein microarrays uses antibodies as probes. They have been widely used in the diagnosis and monitoring of disease but also have applications that extend beyond the field of biomedicine. For instance, Parro et al. (2011) designed and built a series of instruments called SOLID (for "Signs Of Life Detector") for the automatic in situ detection and identification of substances that can be applied in the search for life in extreme environments and planetary exploration (Parro et al., 2011). This kind of microarrays are multiplex immunosensors with antibodies targeting polymeric biomolecules such as lipoteichoic acids, peptidoglycan, DNA, exopolysaccharides, proteins or whole cells, and can be used for biomarker and community profiling in environmental samples or for veterinary and biomedical applications (Palacín et al., 2012).

Although, to the best of our knowledge, protein microarrays have not been used to study phage-microbe interactions, they have been used to address complex aspects of the interaction between human viruses and their target cells. Therefore, why couldn't they be used to study the interactions of phages with 
their host bacterial communities? For instance, virion proteins immobilized on microarrays could be used to "fish" host envelope components involved in phage recognition. Furthermore, immunosensors constructed with antibodies against different phages could be used for monitoring the presence of these phages in environmental samples.

Finally, glycan (or carbohydrate) arrays allow the evaluation, in a high-throughput manner, of interactions between carbohydrates and proteins (including antibodies), viruses and cells (see Liang and Wu, 2009; Rillahan and Paulson, 2011). These arrays can be used to probe hundreds of receptor-ligand interactions in one experiment. They have been applied to the study of the activity of carbohydrate modifying enzymes and the roles of glycan in the detection of diseases such as AIDS and other viral and infectious diseases and in vaccine development. These chips can be built either by immobilizing known glycans or with so-called shotgun glycomics, which uses bulk glycans extracted from the cells of interest (Song et al., 2011).

In 2004, Blixt et al. (2004) published the description of an array of more than 200 carbohydrates representing major glycan structures of glycoproteins and glycolipids. This "glycochip" can be used for profiling the specificity of a diverse range of mammalian, plant, viral and bacterial glycan binding proteins (lectins), as well as of antibodies and intact viruses. Currently, the Consortium for Functional Glycomics has available an array with 610 diverse glycans found in mammalian cells that can be used for a wide range of applications within the biomedical field, such as the profiling of immune response to infection or the glycomics of several diseases of different etiology (www.functionallycomics. org). However, applications of the array extend beyond strictly disease-oriented analyses and have been used, for instance, to unveil interactions between the gut microbiota and the host environment (Garrido et al., 2011).

In a recent pioneering work within the field of phage ecology (Barr et al., 2013), the glycoarray was applied to the analysis of the interactions of bacteriophage and gut mucus. These authors demonstrated that mucus surfaces in metazoans were enriched in bacteriophages compared to the surrounding environments and that this enrichment occurred by binding between mucin glycopetides and immunoglobulin-like domains exposed on the capsid surfaces. Based on the results of their study, the authors proposed the bacteriophage adherence to mucus (BAM) model according to which phages provide a non-host derived antimicrobial defense on the mucosal surfaces of diverse metazoan hosts.

The presence of Ig-like proteins has been found in structural protein of approximately one fourth of sequenced tailed phages where they were proposed to facilitate adherence to bacterial cell surfaces during infection (Fraser et al., 2007). Given the relevance of carbohydrates in the phage-host recognition, it seems feasible that this approach may also be useful within the field of phage biology. For instance, in the above mentioned example of Kunin et al. (2008) the CAP strains detected in the bioreactors in the USA and Australia, although very closely related, presented highly variable extracellular polymeric substances (EPS) gene cassettes. EPS can act as a first line of defense against phage predation and, conversely, lytic bacteriophages can encode strain-specific
EPS degrading polysaccharases (Sutherland, 2001). Conversely, carbohydrate binding and lectin-like domains have frequently been found within the metaviromic islands in marine phages (see the paper by Mizuno et al., 2014). Carbohydrate microarrays could then be a very powerful tool to explore this kind of glycan-mediated phage-microbe interaction.

\section{FINAL REMARKS}

Figure 1 summarizes all the applications of the different kinds of microarrays presented here. Our point has not been to convince readers to use microarrays but to illustrate the power of the combination of several high throughput techniques to unveil relevant aspects of virus-microbe interactions in the past and, hopefully, in the future. We hope that this goal has been accomplished.

\section{ACKNOWLEDGMENTS}

Our current studies with viral microarrays are supported by projects CGL2012-39627-C03-01 (to Josefa Antón) and AYA2011-24803 (to Víctor Parro) of the Spanish Ministry of Science and Innovation, which are co-financed with FEDER support from the European Union.

\section{REFERENCES}

Allen, H. K., and Abedon, S. T. (2013). That's disturbing! An exploration of the bacteriophage biology of change. Front. Microbiol. 4:295. doi: 10.3389/fmicb.2013.00295

Allen, L. Z., Ishoey, T., Novotny, M. A., McLean, J. S., Lasken, R. S., and Williamson, S. J. (2011). Single virus genomics: a new tool for virus discovery. PLoS ONE 6:e17722. doi: 10.1371/journal.pone.0017722

Allen, M. J., and Wilson, W. H. (2008). Aquatic virus diversity accessed through omic techniques: a route map to function. Curr. Opin. Microbiol. 11, 226-232. doi: 10.1016/j.mib.2008.05.004

Barr, J. J., Auro, R., Furlan, M., Whiteson, K. L., Erb, M. L., Pogliano, J., et al. (2013). Bacteriophage adhering to mucus provide a non-host-derived immunity. Proc. Natl. Acad. Sci. U.S.A. 110, 10771-10776. doi: 10.1073/pnas. 1305923110

Ben-Ari, Y., Glick, Y., Kipper, S., Schwartz, N., Avrahami, D., Barbiro-Michaely, E., et al. (2013). Microfluidic large scale integration of viral-host interaction analysis. Lab Chip Miniaturisation Chem. Biol. 13, 2202-2209. doi: $10.1039 / \mathrm{c} 31 \mathrm{c} 00034 \mathrm{f}$

Berard, A., Kroeker, A. L., and Coombs, K. M. (2012). Transcriptomics and quantitative proteomics in virology. Future Virol. 12, 1193-1204. doi: 10.2217/fvl. 12.112

Blixt, O., Head, S., Mondala, T., Scanlan, C., Huflejt, M. E., Alvarez, R., et al. (2004). Printed covalent glycan array for ligand profiling of diverse glycan binding proteins. Proc. Natl. Acad. Sci. U.S.A. 101, 17033-17038. doi: 10.1073/pnas.0407902101

Breitbart, M., Haynes, M., Kelley, S., Angly, F., Edwards, R. A., Felts, B., et al. (2008). Viral diversity and dynamics in an infant gut. Res. Microbiol. 159, 367-373. doi: 10.1016/j.resmic.2008.04.006

Fraser, J. S., Maxwell, K. L., and Davidson, A. R. (2007). Immunoglobulin-like domains on bacteriophage: weapons of modest damage?. Curr. Opin. Microbiol. 10, 382-387. doi: 10.1016/j.mib.2007.05.018

Garrido, D., Kim, J. H., German, J. B., Raybould, H. E., and Mills, D. A. (2011). Oligosaccharide binding proteins from Bifidobacterium longum subsp. infantis reveal a preference for host glycans. PLOS ONE 6:e17315. doi: 10.1371/journal.pone.0017315

Hsu, K. L., and Mahal, L. K. (2009). Sweet tasting chips: microarray-based analysis of glycans. Curr. Opin. Chem. Biol. 13, 427-432. doi: 10.1016/j.cbpa.2009. 07.013

Hynes, A. P., Mercer, R. G., Watton, D. E., Buckley, C. B., and Lang, A. S. (2012). DNA packaging bias and differential expression of gene transfer agent genes within a population during production and release of the Rhodobacter capsulatus gene transfer agent, RcGTA. Mol. Microbiol. 85, 314-325. doi: 10.1111/j.1365-2958.2012.08113.x 
Kunin, V., He, S., Warnecke, F., Peterson, S. B., Garcia Martin, H., Haynes, M., et al. (2008). A bacterial metapopulation adapts locally to phage predation despite global dispersal. Genome Res. 18, 293-297. doi: 10.1101/gr.6835308

Liang, C.-H., and Wu, C.-Y. (2009). Glycan array: a powerful tool for glycomics studies. Expert Rev. Proteomics 6, 631-645. doi: 10.1586/epr.09.82

Lindell, D., Jaffe, J. D., Coleman, M. L., Futschik, M. E., Axmann, I. M., Rector, T., et al. (2007). Genome-wide expression dynamics of a marine virus and host reveal features of co-evolution. Nature 449, 83-86. doi: 10.1038/nature06130

Luke, K., Radek, A., Liu, X., Campbell, J., Uzan, M., Haselkorn, R., et al. (2002). Microarray analysis of gene expression during bacteriophage T4 infection. Virology 299, 182-191. doi: 10.1006/viro.2002.1409

Martínez-García, M., Santos, F., Moreno-Paz, M., Parro, V., and Antón, J. (in press). Unveiling viral-host interactions within the 'microbial dark matter'. Nat. Commun.

Millard, A. D., Zwirglmaier, K., Downey, M. J., Mann, N. H., and Scanlan, D. J. (2009). Comparative genomics of marine cyanomyoviruses reveals the widespread occurrence of Synechococcus host genes localized to a hyperplastic region: implications for mechanisms of cyanophage evolution. Environ. Microbiol. 11, 2370-2387. doi: 10.1111/j.1462-2920.2009.01966.x

Mizuno, C. M., Ghai, R., and Rodriguez-Valera, F. (2014). Evidence for metaviromic islands in marine phages. Front. Microbiol. 5:27. doi: 10.3389/fmicb.2014. 00027

Palacín, A., Gómez-Casado, C., Rivas, L. A., Aguirre, J., Tordesillas, L., Bartra, J., et al. (2012). Graph based study of allergen cross-reactivity of plant lipid transfer proteins (LTPs) using microarray in a multicenter study. PLOS ONE 7:e50799. doi: 10.1371/journal.pone.0050799

Parro, V., de Diego-Castilla, G., Rodriguez-Manfredi, J. A., Rivas, L. A., BlancoLopez, Y., Sebastian, E., et al. (2011). SOLID3: a multiplex antibody microarraybased optical sensor instrument for in situ life detection in planetary exploration. Astrobiology 11, 15-28. doi: 10.1089/ast.2010.0501

Rillahan, C. D., and Paulson, J. C. (2011). Glycan microarrays for decoding the glycome. Annu. Rev. Biochem. 80, 797-823. doi: 10.1146/annurev-biochem061809-152236

Santos, F., Moreno-Paz, M., Meseguer, I., López, C., Rosselló-Mora, R., Parro, V., et al. (2011). Metatranscriptomic analysis of extremely halophilic viral communities. ISME J. 5, 1621-1633. doi: 10.1038/ismej.2011.34

Schena, M., Shalon, D., Davis, R. W., and Brown, P. O. (1995). Quantitative monitoring of gene expression patterns with a complementary DNA Microarray. Science 270, 467-470. doi: 10.1126/science.270.5235.467
Snyder, J. C., Bateson, M. M., Lavin, M., and Young, M. J. (2010). Use of cellular CRISPR (clusters of regularly interspaced short palindromic repeats) spacer-based microarrays for detection of viruses in environmental samples. Appl. Environ. Microbiol. 76, 7251-7258. doi: 10.1128/AEM. 01109-10

Song, X., Lasanajak, Y., Xia, B., Heimburg-Molinaro, J., Rhea, J. M., Ju, H., et al. (2011). Shotgun glycomics: a microarray strategy for functional glycomics. Nat. Methods 8, 85-90. doi: 10.1038/nmeth.1540

Sutherland, I. (2001). Biofilm exopolysaccharides: a strong and sticky framework. Microbiology 147, 3-9.

Uzoma, I., and Zhu, H. (2013). Interactome mapping: using protein microarray technology to reconstruct diverse protein networks. Genomics Proteomics Bioinformatics 11, 18-28. doi: 10.1016/j.gpb.2012.12.005

Waller, A. S., Hug, L. A., Mo, K., Radford, D. R., Maxwell, K. L., and Edwards, E. A. (2012). Transcriptional analysis of a Dehalococcoides-containing microbial consortium reveals prophage activation. Appl. Environ. Microbiol. 78, 1178-1186. doi: 10.1128/AEM.06416-11

Walther, J., Sierocinski, P., and van der Oost, J. (2011). Hot transcriptomics. Archaea 2010, 897585. doi: 10.1155/2010/897585

Wang, D., Coscoy, L., Zylberberg, M., Avila, P. C., Boushey, H. A., Ganem, D., et al. (2002). Microarray-based detection and genotyping of viral pathogens. Proc. Natl. Acad. Sci. U.S.A. 99, 15687-15692. doi: 10.1073/pnas.242579699

Conflict of Interest Statement: The authors declare that the research was conducted in the absence of any commercial or financial relationships that could be construed as a potential conflict of interest.

Received: 10 February 2014; accepted: 16 June 2014; published online: 07 July 2014. Citation: Santos F, Martínez-García M, Parro V and Antón J (2014) Microarray tools to unveil viral-microbe interactions in nature. Front. Ecol. Evol. 2:31. doi: 10.3389/ fevo.2014.00031

This article was submitted to Evolutionary and Genomic Microbiology, a section of the journal Frontiers in Ecology and Evolution.

Copyright (c) 2014 Santos, Martínez-García, Parro and Antón. This is an openaccess article distributed under the terms of the Creative Commons Attribution License (CC BY). The use, distribution or reproduction in other forums is permitted, provided the original author(s) or licensor are credited and that the original publication in this journal is cited, in accordance with accepted academic practice. No use, distribution or reproduction is permitted which does not comply with these terms. 